\title{
Overexpression of CD26/DPPIV in mesothelioma tissue and mesothelioma cell lines
}

\author{
VISHWA JEET AMATYA $^{1}$, YUKIO TAKESHIMA ${ }^{1}$, KEI KUSHITANI ${ }^{1}$, \\ TAKETO YAMADA $^{2}$, CHIKAO MORIMOTO ${ }^{3}$ and KOUKI INAI ${ }^{1}$ \\ ${ }^{1}$ Department of Pathology, Graduate School of Biomedical Sciences, Hiroshima University, Hiroshima; \\ ${ }^{2}$ Department of Pathology, Keio University, Tokyo; ${ }^{3}$ Division of Clinical Immunology, Advanced \\ Clinical Research Center, Institute of Medical Sciences, University of Tokyo, Tokyo, Japan
}

Received July 7, 2011; Accepted August 1, 2011

DOI: $10.3892 / o r .2011 .1449$

\begin{abstract}
Mesothelioma, a highly aggressive cancer with poor prognosis and refractory to currently available therapies show increasing trends of its incidence in Japan and other developing countries. Although surgery is a gold standard for patients with early mesothelioma, most patients with advanced disease are not suitable for surgical resection and have option of palliative chemotherapy alone. One of the new treatment strategies for mesothelioma, the humanized anti-CD26 monoclonal antibody therapy is under development. CD26, a 110-kDa transmembrane glycoprotein with known dipeptidyl peptidase IV activity, plays a role in tumor development and its expression was reported in various human malignancies. This study determined the preliminary selection criteria for humanized monoclonal anti-CD26 antibody therapy. Eighty-one epithelioid (49 differentiated and 32 less differentiated), 34 sarcomatoid, 19 biphasic mesothelioma and 8 mesothelioma cell lines were immunohistochemically examined using 8 different commercially available anti-CD26 antibodies for membranous and cytoplasmic expression. The cytoplasmic expression of CD26 was observed in all histological types of mesothelioma, while the membranous expression of CD26 was found in $88 \%$ of differentiated and $69 \%$ of less differentiated epithelioid mesothelioma, and none of sarcomatoid mesothelioma with anti-CD26 antibodies with rabbit polyclonal anti-DPP4 antibody and similar results were also obtained with goat polyclonal anti-DPP4/CD26 antibody. These antibodies absorbed with soluble human CD26 proteins do not show CD26 expression in mesothelioma tissue, suggesting these two antibodies localize true CD26 protein. Seven mesothelioma cell lines, including sarcomatoid types, also showed membranous expression of CD26 in cellblock preparation.
\end{abstract}

Correspondence to: Dr Vishwa Jeet Amatya, Department of Pathology, Graduate School of Biomedical Sciences, Hiroshima University, 1-2-3 Kasumi, Minami-ku, Hiroshima, 734-8551, Japan E-mail: amatya@hiroshima-u.ac.jp

Key words: CD26, immunohistochemistry, mesothelioma, mesothelioma cell lines
CD26 vector transfection to CD26-negative MSTO-211H cells showed membranous expression of CD26 by flow cytometry, but not in tumor developed in NOD/SCID mice with inoculation of CD26 vector transfected MSTO-211H cells. We found that both rabbit and goat polyclonal antibodies are suitable for immunohistochemical evaluation of membranous expression of CD26 in mesothelioma.

\section{Introduction}

Mesothelioma, primarily associated with exposure to asbestos, causes about 2,500 deaths per year in the United States alone (1). Although the incidence of mesothelioma has been reported as declining in the USA and Canada, a recent study still show a slight increase in death due to mesothelioma in USA (2) and its incidence is rising in Europe, Japan and other developing countries, causing more deaths. The data obtained from the 'Vital Statistics' published by Ministry of Health, Labor and Welfare, Japan indicated that there is an increasing trend in the death due to mesothelioma, from 500 cases in 1995 to 710 cases in 2000, and 911 cases in 2005 (3). It is a highly aggressive cancer with poor prognosis and refractory to currently available therapies $(4,5)$. The conventional modalities in management of mesothelioma by either surgery or radiotherapy and chemotherapy are not very effective, though some of the recent clinical trials of trimodality therapy with neoadjuvant chemotherapy followed by extra-pleural pneumonectomy and radiotherapy have shown few months survival advantage over conventional therapy (6-8). In population-based studies, survival times ranged from 5 months to 13.2 months (9). Although surgery is an option for patients with early-stage mesothelioma, most patients present with advanced locally invasive disease, not amenable to surgical resection. For these patients, the current best treatment is palliative combination chemotherapy with cisplatin and pemetrexed. While this treatment regimen relieves symptoms and has been shown to confer a modest survival benefit (10), median survival is still only 9-12 months from diagnosis.

Therefore, the new treatment strategies for management of mesothelioma are an urgent need in the coming years. Various therapeutic monoclonal antibodies have emerged as new treatment modality in the treatment of cancers. Trastuzumab 
Table I. Source of antibodies.

\begin{tabular}{lllc}
\hline Source/manufacturer & Catalogue no. & \multicolumn{1}{c}{ Host/clone } & Primary choice of application \\
\hline Santa Cruz & SC-9153 & Rabbit/polyclonal & WB, IP, IF, ELISA \\
MBL International & DO68-1 & Mouse (Clone 44-4) & FC, IHC \\
Abcam & Ab61825 & Rabbit/polyclonal & IHC-Paraffin \\
Lifespan Biosciences (MBL International) & LS-A8060 & Rabbit/polyclonal & IHC-Paraffin \\
Acris Antibodies GmbH & AP07044PU-N & Rabbit/polyclonal & IHC-Paraffin \\
Imgenex & IMG72095 & Rabbit/polyclonal & IHC-Paraffin \\
Novus Biological & NB100-59021 & Rabbit/polyclonal & IHC-Paraffin \\
R\&D Systems & AF1180 & Goat/polyclonal & WB, ELISA, IHC
\end{tabular}

WB, Western blotting; IP, immunoprecipitation; IF, immunofluorescence; ELISA, enzyme linked immunosorbent assay; FC, flow cytometry; IHC, immunohistochemistry.

targeting ErbB2 in breast cancer, cetuximab targeting EGFR or bevacizumab targeting VEGF in colorectal or lung cancers have already entered into standard clinical practice (reviewed in ref. 11). Humanized anti-CD26 monoclonal antibody having inhibitory effect on malignant mesothelioma cell growth in vitro and in vivo experiments has been reported (12), one of the a promising new developments for the antibody-based treatment of mesothelioma $(12,13)$.

CD26 is a widely distributed $110-\mathrm{kDa}$ transmembrane glycoprotein with known dipeptidyl peptidase IV activity in its extracellular domain $(14,15)$. It is discovered as a lymphocyte cell surface antigen. It increases during T-cell activation as an essential role in the immune regulation and functions as a regulator of chemokine function. It has been reported to be expressed in various other tissues, placenta, kidney, lung, brain, liver, endothelium, and intestines (16-19). CD26 also plays a role in tumor development and its expression was reported in various human malignancies, mainly cancers of thyroid, breast, prostate, and ovary $(13,20)$. We immunohistochemically evaluated the expression of CD26 in mesothelioma in determining the proper selection criteria of the mesothelioma patients for humanized monoclonal anti-CD26 antibody treatment.

\section{Materials and methods}

Tissue samples and mesothelioma cell lines. The formalinfixed paraffin-embedded (FFPE) tissue materials from mesothelioma cases were obtained from the tissue archives of the Department of Pathology, Hiroshima University. They were derived from patients who had undergone surgical resection or autopsy. The clinicoradiological data were retrieved from the hospital records. The microscopic slides were reviewed and reclassified using the current histological classification of tumors of lung and pleura, World Health Organization 2004. Epithelioid mesothelioma was further sub-divided into two subtypes, i.e., 'differentiated' type (EM-D) and 'lessdifferentiated' type (EM-LD) based on the morphology of 'papillo-tubular structures' as an indicator of differentiation. Thus, EM-D were epithelioid mesothelioma showing papillotubular pattern, micropapillary pattern, and/or microcystic pattern and EM-LD showing solid nest, trabecular pattern, signet-ring cell-like appearance and/or single cell infiltration pattern. One representative tissue block was used for immunohistochemical confirmation of mesothelioma using positive and negative antibody panels mentioned in our previous publications (21-25). This study consisted of 81 cases of epithelioid mesothelioma (EM) (61\%), 34 cases of sarcomatoid mesothelioma (SM) (25\%) and 19 cases of biphasic mesothelioma (BM) (14\%). The mean age of patients was 61.9 years (23-87 years), and male/female ratio was 6:1. Forty-nine cases of epithelioid mesothelioma were differentiated type (EM-D) showing histological patterns of tubulopapillary, microcystic, or myxoid growth and 32 cases were less differentiated type (EM-LD) showing histological patterns of solid-sheet with polygonal, pleomorphic or bizarre giant cells. The study was carried out in accordance with the Ethical Guidelines for Human Genome/Gene Research of the Japanese Government.

Six epithelial type (epithelioid) and 2 fibroblastic type (sarcomatoid) mesothelioma cell lines were purchased either from Riken, Tokyo, Japan (ACC-MESO1, ACC-MESO4, HMMME) or American Type Culture Collection, Manassas, VA, USA (NCI-H226, NCI-H2452, MSTO-211H, NCI-H28, NCI-H2052). All mesothelioma cell lines were maintained in RPMI-1640 media with $10 \%$ fetal bovine serum, and $1 \%$ kanamycin, and $1 \%$ fungizone (all purchased from Gibco) at $37^{\circ} \mathrm{C}$ with $5 \% \mathrm{CO}_{2}$.

Each of mesothelioma cell lines was cultured for 6-10 days on laminin-coated wells of Lab-Tek II chamber slide system (Thermo Fisher Scientific, Tokyo, Japan) and $100 \mathrm{~mm}$ CellBind culture dish (Corning Life Sciences, Corning, NY). Cells in chamber slides were directly fixed with $10 \%$ buffered formalin for $10 \mathrm{~min}$. Cells in culture dish were subjected to cell-block preparation using glucomannan (HoldGel 110, EBIS1 cell-block construction kit, Asia Kizai, Tokyo, Japan) according to the manufacturer's protocol.

CD 26 immunohistochemistry. CD 26 immunohistochemistry was performed in 3- $\mu \mathrm{m}$-tissue sections prepared from formalin-fixed paraffin-embedded tissue blocks in APS-coated slides. Sections were deparaffinized by four changes of xylene, and rehydrated with graded series of ethanol. Endogenous peroxidase was inactivated by treatment with $0.3 \% \mathrm{H}_{2} \mathrm{O}_{2}$ in PBS for $30 \mathrm{~min}$. The tissue sections were then incubated 


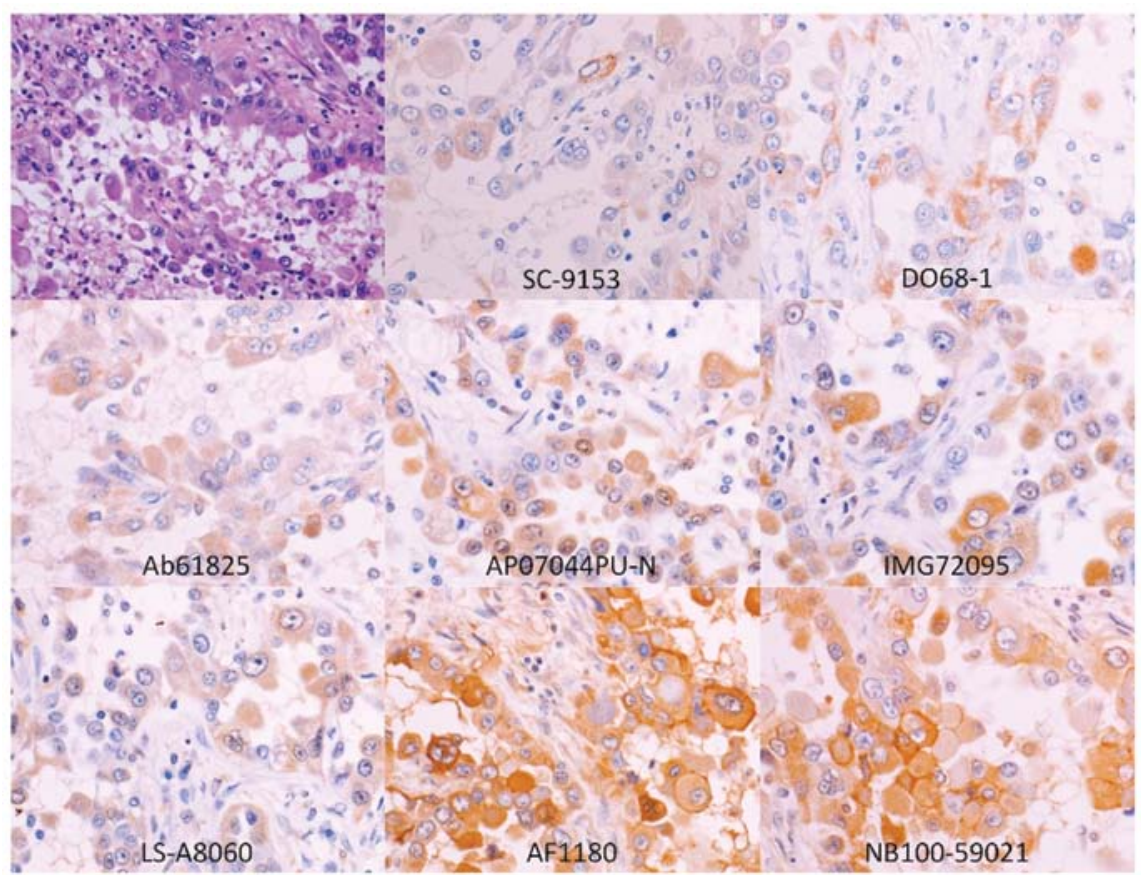

Figure 1. CD26 expression in epithelioid mesothelioma with various antibodies. Cytoplasmic expression of CD26 is observed with all the 8 anti-CD26/DPP4 antibodies studied. The membranous expression of CD26 is detected with only CD26-NB100/59021 and CD26-AF1180 antibodies.

with commercially available anti-CD26/DPPIV antibodies purchased from various sources (Table I) at $4^{\circ} \mathrm{C}$ overnight in a humidified chamber. The reaction was visualized using the Histofine Simple Stain kits (Nichirei Biosciences, Tokyo, Japan) and diaminobenzidine (Dojindo Laboratories, Tokyo, Japan) as the chromogen. The tissue sections were counterstained for nucleus with Mayer's haematoxylin. Similar immunohistochemical procedure was carried out with the omission of the primary antibody as a negative control. CD26 immunohistochemical procedure was also performed in mesothelioma cells on chamber slides and sections prepared from cell line blocks.

The membranous and cytoplasmic expression of CD26 was semi-quantitatively analyzed. The scoring system was based mainly on membranous staining: 0 , no membranous staining of tumor cells; $1+$, membrane staining in up to $25 \%$ of tumor cells; +2 , membrane staining observed in $26-50 \%$ of tumor cells; +3 , membrane staining in $>50 \%$ of tumor cells.

Absorption study using soluble human CD26 proteins. Absorption study by soluble CD26 antigen was conducted to validate whether signal by immunohistochemistry using rabbit anti-human DPP4 polyclonal antibody (NB100-59021, Novus Biologicals, Littleton, CO, USA, hereafter we call as CD26-NB100/59021) or goat anti-human DPPIV/CD26 polyclonal antibody (AF1180, R\&D Systems Inc., Minneapolis, MN, USA, hereafter we call as CD26-AF1180) corresponds to true CD26 localization or not. Two $\mu \mathrm{g}$ of rabbit antihuman CD26-NB100/59021 or goat anti-human polyclonal antibody (CD26-AF1180) was incubated with $5 \mu \mathrm{g}$ of recombinant soluble human CD26 proteins (produced by CHO cells, provided by Dr Kei Ohnuma, Tokyo University) overnight at $4^{\circ} \mathrm{C}$. The solution was then centrifuged at 5,000 $\mathrm{x}$ for $10 \mathrm{~min}$ and the pellet was discarded. The immunohistochemistry using this absorbed primary antibody as primary antibody was carried out as described above.

CD26 transfection into CD26-negative MSTO-211H mesothelioma cells. MSTO-211H cells, which do not express CD26, were cultured as described above. MSTO-211H cells adhered to culture dish were transfected with a full-length CD26 subcloned retroviral shuttle plasmid pLNCX2 vector (Clontech Inc., Mountain View, CA, USA) using the Lipofectin reagent (Invitrogen). Fresh media with 10\% FBS was replenished $18 \mathrm{~h}$ after transfection and cells were harvested after $48 \mathrm{~h}$.

The cells were stained with anti-human CD26 monoclonal antibody (BA5, Phycoerythrin-conjugated, diluted 1:100, Dako) for analysis with a flow cytometer (EPICS XL-MCL, Beckman Coulter, Hialeah, USA). Normal mouse IgG2a (Sigma-Aldrich) was used as a control.

Inoculation of MSTO-211H cells into NOD/SCID mice. All in vivo studies were approved by the Animal Care and Use Committee of Keio University. NOD/SCID mice were bred and maintained in Department of Pathology, Keio University. The human CD26 vector transfected-MSTO-211H cells were inoculated subcutaneously into the left flank of 6-week old female NOD/SCID mice. The resected subcutaneous tumor was fixed with $10 \%$ formalin and embedded in paraffin for immunohistochemical study.

\section{Results}

CD26 expression in mesothelioma tissue. The immunohistochemical staining of CD26 expression was carried out with 8 different commercially available antibodies against CD26/ DPP4. Using any of these antibodies, cytoplasmic expression of CD26 (cCD26) was observed in all mesothelioma cases (Fig. 1). 
Table II. CD26 immunohistochemical score in mesothelioma. ${ }^{\mathrm{a}}$

\begin{tabular}{|c|c|c|c|c|c|c|c|c|}
\hline & \multicolumn{4}{|c|}{ CD26-NB100/59021 expression } & \multicolumn{4}{|c|}{ CD26-AF1180 expression } \\
\hline & Score 0 & Score 1 & Score 2 & Score 3 & Score 0 & Score 1 & Score 2 & Score 3 \\
\hline \multicolumn{9}{|l|}{ EM-D } \\
\hline Cytoplasmic & 0 & 3 & 12 & 34 & 0 & 2 & 12 & 35 \\
\hline Membranous & 6 & 12 & 16 & 15 & 6 & 16 & 19 & 8 \\
\hline \multicolumn{9}{|l|}{ EM-LD } \\
\hline Cytoplasmic & 0 & 2 & 11 & 19 & 0 & 0 & 15 & 17 \\
\hline Membranous & 10 & 7 & 11 & 4 & 10 & 7 & 12 & 3 \\
\hline \multicolumn{9}{|c|}{ BM-epithelioid component } \\
\hline Cytoplasmic & 0 & 1 & 8 & 9 & 0 & 1 & 6 & 11 \\
\hline Membranous & 1 & 4 & 8 & 5 & 4 & 6 & 6 & 2 \\
\hline \multicolumn{9}{|c|}{ BM-sarcomatoid component } \\
\hline Cytoplasmic & 0 & 3 & 15 & 0 & 0 & 4 & 13 & 1 \\
\hline Membranous & 18 & 0 & 0 & 0 & 18 & 0 & 0 & 0 \\
\hline \multicolumn{9}{|c|}{ Sarcomatoid mesothelioma } \\
\hline Cytoplasmic & 0 & 9 & 21 & 5 & 0 & 12 & 7 & 6 \\
\hline Membranous & 35 & 0 & 0 & 0 & 35 & 0 & 0 & 0 \\
\hline
\end{tabular}

aDetailed data available online (http://home.hiroshima-u.ac.jp/byouri2/JP/Supple.html). EM-D, epithelioid mesothelioma-differentiated type; EM-LD, epithelioid mesothelioma-less differentiated type; BM, biphasic mesothelioma.

Table III. Comparison of CD26 immunohistochemical score of CD26 expression with two antibodies.

\begin{tabular}{rrrrrr}
\hline & & \multicolumn{4}{c}{ NB100-59021 } \\
\cline { 3 - 6 } & & 0 & $1+$ & $2+$ & $3+$ \\
\hline \multirow{2}{*}{ AF1180 } & 0 & 13 & 5 & 2 & 0 \\
& $1+$ & 4 & 14 & 10 & 1 \\
& $2+$ & 0 & 4 & 21 & 12 \\
& $3+$ & 0 & 0 & 2 & 11 \\
\hline
\end{tabular}

p-value $<0.0001$.

However, the membranous expression of CD26 (mCD26) could not be detected with SC-9153, DO-68, Ab61825, LS-A8060, AP01046PU-N, and IMG72095 antibodies. The membranous expression of CD26 in mesothelioma was only detected using either CD26-NB100/59021 or CD26-AF1180 antibodies.

CD26 expression was found in almost all cases of mesothelioma with membranous and/or cytoplasmic pattern using CD26-NB100/59021 or CD26-AF1180 antibody (Table II). The membranous expression patterns between these two antibodies were very similar (Fig. 1 and Table III). Using CD26-NB100/59021 antibody, the membranous expression of CD26 was found in 65 of $81(80 \%)$ epithelioid mesotheliomas (Fig. 2, Table II), and 17 of 18 (89\%) epithelioid component of biphasic mesothelioma. The membranous expression of CD26 was not found in sarcomatoid mesothelioma or sarcomatoid
Table IV. Immunohistochemical score of membranous CD26 expression.

\begin{tabular}{lccccc}
\hline & \multicolumn{5}{c}{ Immunohistochemical score (\%) } \\
\cline { 2 - 6 } & Total & 0 & $1+$ & $2+$ & $3+$ \\
\hline EM-D & 49 & $6(12.2)$ & $12(24.5)$ & $16(32.7)$ & $15(30.6)$ \\
EM-LD & 32 & $10(31.2)$ & $7(21.9)$ & $11(34.4)$ & $4(12.5)$ \\
BM (E) & 18 & $1(5.5)$ & $4(22.2)$ & $8(44.5)$ & $5(27.8)$ \\
\hline EM-D, epithelioid mesothelioma differentiated type; EM-LD, epi- \\
thelioid mesothelioma-less differentiated type. BM (E), epithelioid \\
component of biphasic mesothelioma.
\end{tabular}

component of biphasic mesothelioma (Fig. 2, Table II). Fifteen of 49 cases (31\%) of EM-D showed mCD26 expression in $>50 \%$ of tumor cells, 16 cases (33\%) in $26-50 \%$ of tumor cells, and only 12 cases $(25 \%)$ showed $<25 \%$ of tumor cells (Table IV). Four of 32 cases (12\%) of EM-LD showed mCD26 expression in $>50 \%$ of tumor cells, 11 cases $(34 \%)$ in $26-50 \%$ of tumor cells, and only 7 cases $(22 \%)$ showed $<25 \%$ of tumor cells (Table IV). Five of 18 cases (27\%) of epithelioid component of $\mathrm{BM}$ showed mCD26 expression in $>50 \%$ of tumor cells, 8 cases $(44 \%)$ in $26-50 \%$ of tumor cells, and only 4 cases $(22 \%)$ showed $<25 \%$ of tumor cells (Table IV).

CD26 expression in mesothelioma cell lines. Seven out of 8 mesothelioma cell lines showed CD26 expression. MSTO$211 \mathrm{H}$ mesothelioma cell line had no expression of CD26. The 


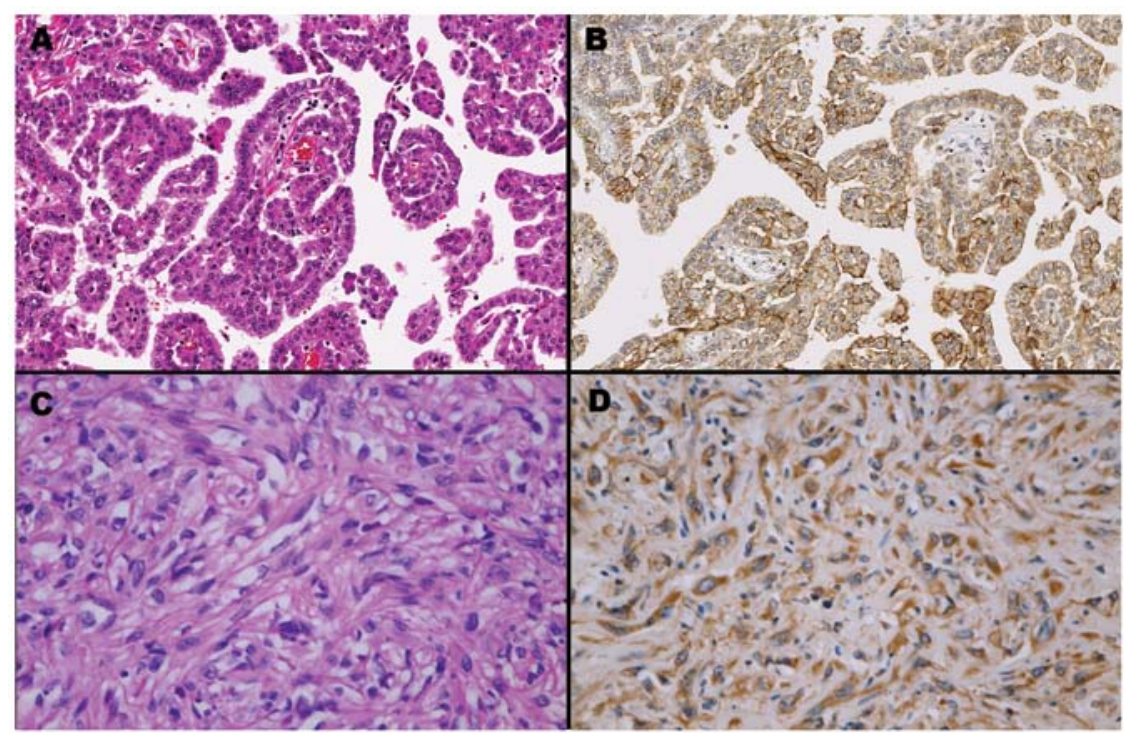

Figure 2. CD26 expression in mesothelioma using CD26-NB100/59021 antibody. Epithelioid mesothelioma differentiated type (A and B), shows membranous expression of CD26 and sarcomatoid mesothelioma (C and D) shows only cytoplasmic expression with inconspicuous membranous expression of CD26. (A and C) Hematoxylin eosin stain; (B and D) CD26 immunohistochemistry.
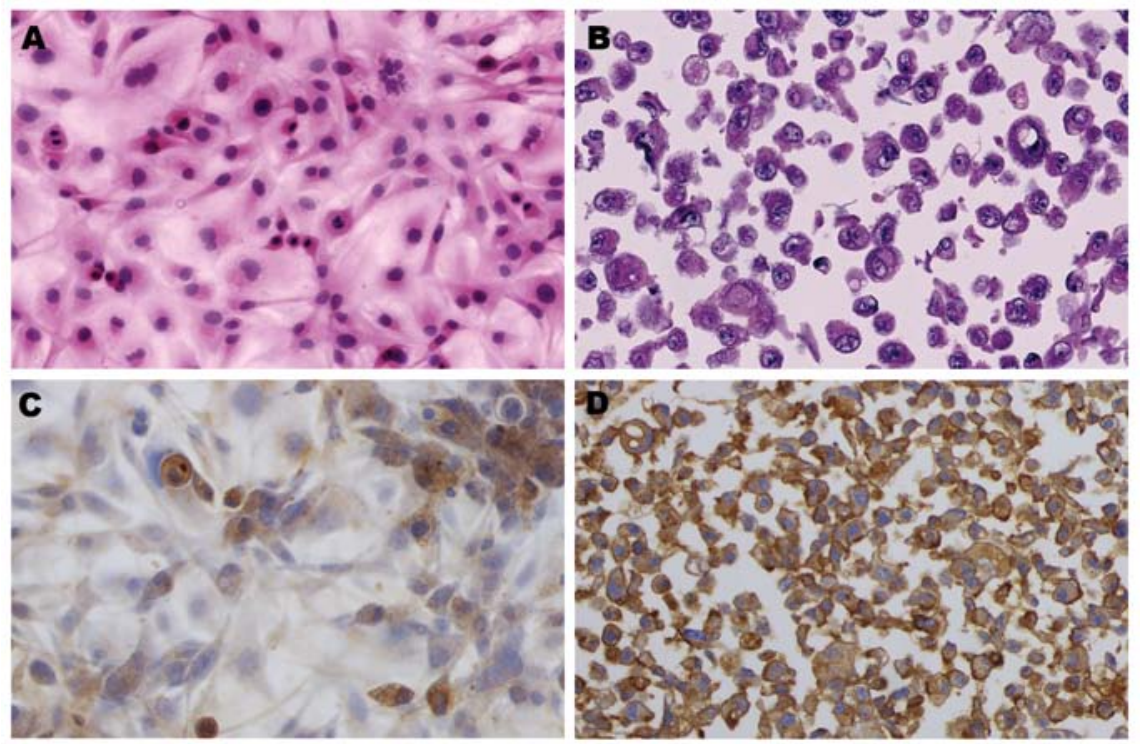

Figure 3. CD 26 expression in mesothelioma cell line. Mesothelioma cell line ACC-MESO-1, shows spindle cell growth in culture dish (A) and no membranous expression pattern of CD26 is detected (C). However, the cellblock preparation (B) from the same cell line shows membranous expression pattern of CD26 in tumor cells (D).

tumor cells derived from sarcomatoid mesothelioma when grown in chamber slides showed morphologically spindleshaped tumor cells (Fig. 3A), and had primarily cytoplasmic CD26 expression pattern with very few cells showing weak membranous expression (Fig. 3B). The cellblock preparations from the same mesothelioma cells, which transformed to round to oval cells due to trypsinization, showed prominent cell membrane (Fig. 3C). In these preparations, mCD26 expression was clearly evident (Fig. 3D).

Validation of polyclonal antibodies for true localization of CD26. Immunohistochemistry using either absorbed primary antibody of rabbit anti-human CD26 polyclonal antibody
(CD26-NB100/59021) or goat anti-human CD26 polyclonal antibody (R\&D, AF1180) showed disappearance of CD26 expression in mesothelioma tissue (Fig. 4), suggesting these two antibodies localizes true CD26 protein.

CD26 expression after CD26 vector transfection into mesothelioma cells and tumor development in NOD/SCID mice. MSTO-211H cells, which primarily do not express CD26, showed membranous expression of CD26 after transfected with a full-length CD26 subcloned retroviral shuttle plasmid pLNCX2 vector by flow cytometric analysis (Fig. 5A).

NOD/SCID mice after inoculation of CD26 vector transfected MSTO-211H cells developed mesothelioma. This tumor 

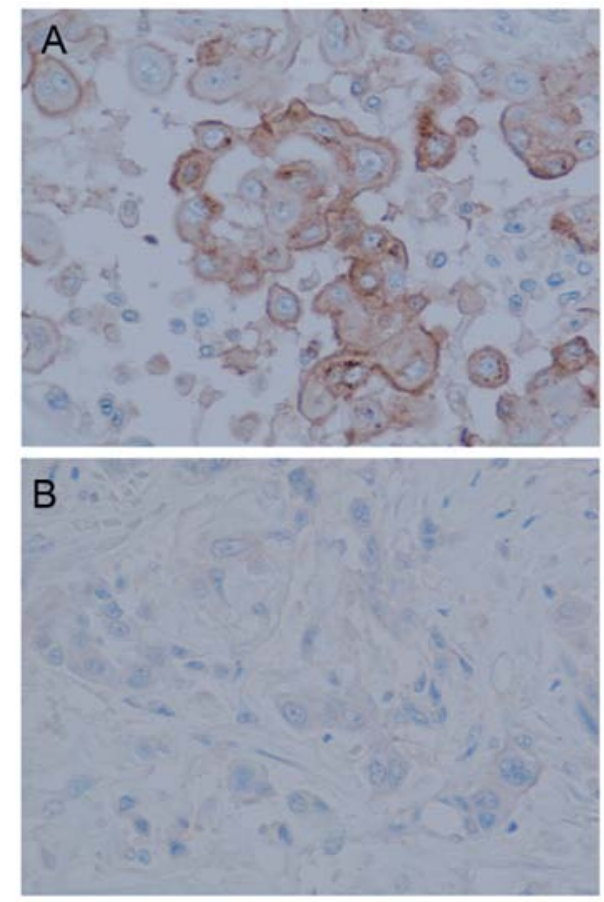

Figure 4. Absorbed CD26 reactions after soluble CD26 antigens in immunohistochemistry. The absorbed CD26 antibody is rabbit anti-human CD26 polyclonal antibody (CD26-NB100/59021) was incubated with recombinant soluble human $\mathrm{CD} 26$ proteins produced by $\mathrm{CHO}$ cells. CD26 immunoreactivity observed with anti-CD26 antibody (A) is not detected with absorbed CD26 antibody (B).

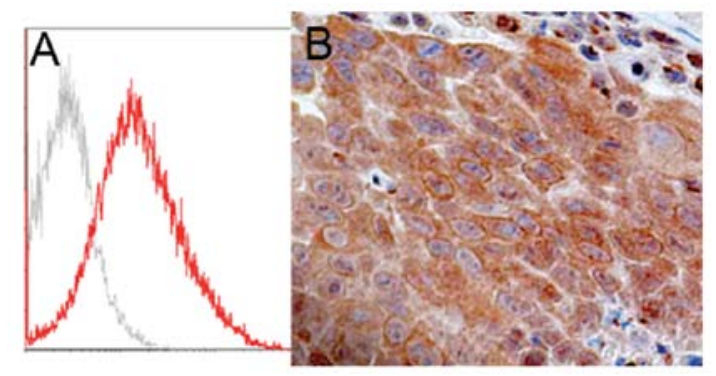

Figure 5. (A) CD26 vector-transfected MSTO-211H cells without fixation showing membranous expression of CD26 by flow cytometric analysis. Red curve, anti-CD26 antibody (phycoerythrin-conjugated monoclonal antibody, BA5); gray curve, control mouse IgG2a. (B) CD26 expression in human CD26 expression vector-transfered MSTO-211H cells. CD26 expression is mainly in the cytoplasm of mesothelioma developed after human CD26 vector-transfected MSTO-211H cells were inoculated into NOD/SCID mice. (Immunohistochemistry with goat anti-human CD26 polyclonal antibody (CD26-AF1180).

showed cytoplasmic expression of CD26. No membranous expression of CD26 could be observed (Fig. 5B).

\section{Discussion}

Various unconjugated or immuno-conjugated monoclonal antibody-based therapies have been developed and already established as a new treatment modality in lymphomas, breast, lung, or colorectal cancers. Anti-CD20 antibody (rituximab) in lymphomas, anti-HER 2 antibody (trastuzumab) in breast cancer, anti-EGFR antibody (cetuximab) or anti-VEGF (beva- cizumab) in colorectal or lung cancers have entered standard clinical practice (reviewed in ref. 11). Other antibodies like MDX-010 humanized mAb (anti-CTLA-4 antibody), which inhibit T-cell activation, are also supposed to be promising (26). The possibility of humanized anti-CD26 monoclonal antibody treatment for its inhibitory effect has been reported on malignant mesothelioma cell lines in vitro and in vivo experiments (12). This is the new promising strategy in antibody-based treatment of mesothelioma $(12,13)$.

The success of all antibody-based treatments of cancer largely depends on the proper selection of the cases. One such selection criterion is by the immunohistochemical expression in the tumor cells. For example, the selection of breast cancer patients for anti-HER2 antibody therapy depends upon the HER-2 expression in the tumor cells of those patients using immunohistochemical study. All breast cancers do not express HER-2 when analyzed by immunohistochemical staining alone and many cases do need further analysis of HER-2 expression by fluorescence in situ hybridization (FISH). The interpretation manual has been developed for the management of such treatment (27). The same rule should be applied to humanized monoclonal CD26 antibody therapy to mesothelioma and proper guidelines for selection of mesothelioma cases are required. We have, in this study, analyzed various commercially available antibodies to detect an antibody with reproducibility in correct interpretation of CD26 expression in the archived mesothelioma tissue and/or mesothelioma cell lines. Out of more than 50 different commercially available antibodies, we have selected the antibodies available in the past and newly developed antibodies available recently for immunohistochemical staining in FFPE sections. We found CD26-NB100/59021 antibody from Novus Biologicals or AF1180 from R\&D systems are equally good for interpretation of membranous CD26 expression. We also validated these two antibodies for true localization of CD26 protein by absorption study using soluble human CD26 proteins.

The morphology of mesothelioma is generally found to be an important prognostic factor, epithelioid mesothelioma cases show better survival than sarcomatoid mesothelioma cases $(28,29)$. In the present study, none of sarcomatoid mesothelioma expressed membranous CD26 in the tumor cells. The reason for this may be due to the morphological characteristics of sarcomatoid mesothelioma, primarily spindled shape. This can be better explained by our findings of CD26 expression in mesothelioma cell lines. The mesothelioma cells grow as spindled cells in culture medium with inconspicuous cell membrane. This can be even observed in some of the mesothelioma cell lines derived from epithelioid mesothelioma cases. However, the same cells, when treated with trypsin, showed round to oval cells with distinct cell membrane. In this study, we found membranous CD26 expression in these mesothelioma cells. In addition, the mesothelioma cells, when analyzed by flow cytometry to detect expression of cell surface antigen, clearly showed CD26 expression. On the other hand, it can also be postulated that the development of sarcomatoid mesothelioma is by epithelial-mesenchymal transition from epithelioid mesothelioma. The concept of epithelial-mesenchymal transition was recognized at first as a central differentiation process in early embryogenic morphogenesis (30). The coordinated molecular and cellular changes defined as the reductions in 
cell-cell adhesion, apical-basolateral polarity, and epithelial markers, as well as the acquisition of motility, spindle-cell shape, and mesenchymal markers. So, CD26 expression, which is prominent in epithelioid mesothelioma, more in differentiated type than less-differentiated type may have been lost after transition into the sarcomatoid mesothelioma, thus showing only cytoplasmic CD26 expression. Our CD26 vector transfected MSTO-211H cells, when inoculated to NOD/ SCID mice, show only cytoplasmic expression though surface antigen is clearly expressed in flow cytometric analysis. The detection of surface CD26 by flow cytometry can be another method of analyzing mCD26 expression in pleural fluid from mesthelioma patients.

In conclusion, we found overexpression of CD26 in mesothelioma tissue and cell lines. By using NB100-59021 or AF1180 antibody, immunohistochemical evaluation of membranous CD26 expression in human mesothelioma tissues is possible for selecting mesothelioma cases for humanized monoclonal anti-CD26 antibody treatment. Sarcomatoid mesothelioma in FFPE which do not show membranous CD26 expression, though existence of membranous expression in mesothelioma cell blocks suggest other method to evaluate CD26 expression such as flow cytometry is needed for selecting these cases for humanized monoclonal anti-CD26 antibody therapy.

\section{Acknowledgements}

The authors thank Ms. Yuka Fukushima for her excellent technical assistance, Ms. Keiko Honda and Naomi Fukuhara for official assistance. This research was supported by the program for promotion of fundamental studies in Health Sciences of the National Institute of Biomedical Innovation (C.M.).

\section{References}

1. Weill H, Hughes JM and Churg AM: Changing trends in US mesothelioma incidence. Occup Environ Med 61: 438-441, 2004

2. Malignant mesothelioma mortality-United States, 1999-2005. MMWR Morb Mortal Wkly Rep 58: 393-396, 2009.

3. Takeshima Y, Inai K, Amatya VJ, et al: Accuracy of pathological diagnosis of mesothelioma cases in Japan: clinicopathological analysis of 382 cases. Lung Cancer 66: 191-197, 2009.

4. Pass HI, Vogelzang NJ, Hahn S and Carbone M: Malignant pleural mesothelioma. Curr Proble Cancer 28: 93-174, 2004.

5. Robinson BW and Lake RA: Advances in malignant mesothelioma. N Engl J Med 353: 1591-1603, 2005.

6. Vogelzang NJ, Rusthoven JJ, Symanowski J, et al: Phase III study of pemetrexed in combination with cisplatin versus cisplatin alone in patients with malignant pleural mesothelioma. J Clin Oncol 21: 2636-2644, 2003.

7. Krug L, Pass H, Rusch V, et al: Multicenter phase II trial of neoadjuvant pemetrexed plus cisplatin dfollowed by extrapleural pneumonectomy and radiation for malignant pleural mesothelioma. J Clin Oncol 18: 3007-3013, 2009.

8. Weder W, Stahel R, Bernhard J, et al: Multicenter trial of neoadjuvant chemotherapy followed by extrapleural pneumonectomy in malignant pleural mesothelioma. Ann Oncol 18: 1196-1202, 2007.

9. Montanaro F, Rosato R, Gangemi M, et al: Survival of pleural malignant mesothelioma in Italy: a population-based study. Int J Cancer 124: 201-207, 2009.

10. Marinaccio A, Montanaro F, Mastrantonio M, et al: Predictions of mortality from pleural mesothelioma in Italy: a model based on asbestos consumption figures supports results from ageperiod-cohort models. Int J Cancer 115: 142-147, 2005.

11. Adams G and Weiner L: Monoclonal antibody therapy of cancer. Nat Biotechnol 23: 1147-1157, 2005.
12. Inamoto $\mathrm{T}$, Yamada $\mathrm{T}$, Ohnuma $\mathrm{K}$, et al: Humanized anti-CD26 monoclonal antibody as a treatment for malignant mesothelioma tumors. Clin Cancer Res 13: 4191-4200, 2007.

13. Thompson MA, Ohnuma K, Abe M, Morimoto C and Dang NH: CD26/dipeptidyl peptidase IV as a novel therapeutic target for cancer and immune disorders. Mini Rev Med Chem 7: 253-273, 2007.

14. Torimoto Y, Dang N, Tanaka T, Prado C, Schlossman S and Morimoto C: Biochemical characterization of CD26 (dipeptidyl peptidase IV): functional comparison of distinct epitopes recognized by various anti-CD26 monoclonal antibodies. Mol Immunol 29: 183-192, 1992.

15. Morimoto C, Torimoto Y, Levinson G, et al: 1F7, a novel cell surface molecule, involved in helper function of CD4 cells. J Immunol 143: 3430-3439, 1989.

16. Abbott CA, Baker E, Sutherland GR and McCaughan GW: Genomic organization, exact localization, and tissue expression of the human CD26 (dipeptidyl peptidase IV) gene Immunogenetics 40: 331-338, 1994.

17. Morrison ME, Vijayasaradhi S, Engelstein D, Albino AP and Houghton AN: A marker for neoplastic progression of human melanocytes is a cell surface ectopeptidase. J Exp Med 177: 1135-1143, 1993

18. Stange T, Kettmann U and Holzhausen HJ: Immunoelectron microscopic demonstration of the membrane proteases aminopeptidase N/CD13 and dipeptidyl peptidase IV/CD26 in normal and neoplastic renal parenchymal tissues and cells. Eur J Histochem 44: 157-164, 2000.

19. Su A, Wiltshire T, Batalov S, et al: A gene atlas of the mouse and human protein-encoding transcriptomes. Proc Natl Acad Sci USA 101: 6062-6067, 2004.

20. Havre P, Abe M, Urasaki Y, Ohnuma K, Morimoto C and Dang N: The role of CD26/dipeptidyl peptidase IV in cancer. Front Biosci 13: 1634-1645, 2008.

21. Kushitani K, Takeshima Y, Amatya VJ, Furonaka O, Sakatani A and Inai K: Immunohistochemical marker panels for distinguishing between epithelioid mesothelioma and lung adenocarcinoma. Pathol Int 57: 190-199, 2007.

22. Takeshima Y, Amatya VJ, Kushitani K and Inai K: A Useful antibody panel for differential diagnosis between peritoneal mesothelioma and ovarian serous carcinoma in Japanese cases. Am J Clin Pathol 130: 771-779, 2008.

23. Kushitani K, Takeshima Y, Amatya VJ, Furonaka O, Sakatani A and Inai K: Differential diagnosis of sarcomatoid mesothelioma from true sarcoma and sarcomatoid carcinoma using immunohistochemistry. Pathol Int 58: 75-83, 2008.

24. Takeshima Y, Amatya VJ, Kushitani K, Kaneko M and Inai K: Value of immunohistochemistry in the differential diagnosis of pleural sarcomatoid mesothelioma from lung sarcomatoid carcinoma. Histopathology 54: 667-676, 2009.

25. Amatya VJ, Takeshima Y, Kohno H, et al: Caveolin-1 is a novel immunohistochemical marker to differentiate epithelioid mesothelioma from lung adenocarcinoma. Histopathology 55: 10-19, 2009.

26. Sanderson K, Scotland R, Lee P, et al: Autoimmunity in a phase I trial of a fully human anti-cytotoxic T-lymphocyte antigen-4 monoclonal antibody with multiple melanoma peptides and Montanide ISA 51 for patients with resected stages III and IV melanoma. J Clin Oncol 23: 741-750, 2005.

27. Wolff A, Hammond M, Schwartz J, et al: American Society of Clinical Oncology/College of American Pathologists guideline recommendations for human epidermal growth factor receptor 2 testing in breast cancer. Arch Pathol Lab Med 131: 18-43, 2007.

28. Edwards J, Abrams K, Leverment J, Spyt T, Waller D and O'Byrne K: Prognostic factors for malignant mesothelioma in 142 patients: validation of CALGB and EORTC prognostic scoring systems. Thorax 55: 731-735, 2000.

29. Antman K, Shemin R, Ryan L, et al: Malignant mesothelioma: prognostic variables in a registry of 180 patients, the DanaFarber Cancer Institute and Brigham and Women's Hospital experience over two decades, 1965-1985. J Clin Oncol 6: 147-153, 1988.

30. Shook D and Keller R: Mechanisms, mechanics and function of epithelial-mesenchymal transitions in early development. Mech Dev 120: 1351-1383, 2003. 